\title{
Enhanced transmission through arrays of subwavelength holes in gold films coated by a finite dielectric layer
}

Sanshui Xiao

sanshui.xiao@mic.dtu.dk

Niels Asger Mortensen nam@mic.dtu.dk

\section{Min Qiu}

\begin{abstract}
MIC - Department of Micro and Nanotechnology, NanoDTU, Technical University of Denmark, DK2800 Kongens Lyngby, Denmark

MIC - Department of Micro and Nanotechnology, NanoDTU, Technical University of Denmark, DK2800 Kongens Lyngby, Denmark

Laboratory of Optics, Photonics and Quantum Electronics, Department of Microelectronics and Applied Physics, Royal Institute of Technology (KTH), Electrum 229, 16440 Kista, Sweden.
\end{abstract}

Enhanced transmissions through a gold film with arrays of subwavelength holes are theoretically studied, employing the rigid full vectorial three dimensional finite difference time domain method. Influence of air-holes shape to the transmission is firstly studied, which confirms two different resonances attributing to the enhanced transmission: the localized waveguide resonance and periodic surface plasmon resonances. For the film coated with dielectric layers, calculated results show that in the wavelength region of interest the localized waveguide resonant mode attributes to sensing rather than the periodic gold-glass surface plasmon mode. Although the detected peak is fairly broad and the shift is not too pronounced, we emphasize the contribution for sensing from the localized waveguide resonant mode, which may opens up new ways to design surface plasmon based sensors. [D01: 10.2971/je0s.2007.07009]

Keywords: transmission, surface plasmon, sensor

\section{INTRODUCTION}

In the recent years, the demonstration of a strong and unexpected enhancement of light transmission through arrays of subwavelength holes has generated numerous experimental and theoretical work [1]-[9]. Although there are still continuing discussions about the physical mechanisms responsible for the extraordinary transmission, many researchers show that the enhanced transmission is attributed to the resonances. A surface plasmon (SP) is a collective oscillation of free electrons inside a metal-dielectric surface. Strong confinement of plasmonic waves near the metal surface provides possibility for enhancing interactions with an analyte layer and thus for efficient sensing of surface binding events. Surface plasmon (SP) sensors are widely used in chemical and biological research [10]. Conventional SP sensors, employing Kretschmann configuration, usually operate in SP-based attenuation total internal refraction. Recently, there has been a growing interest in surface plasmon resonance (SPR) sensing of biochemicals using nanohole arrays [11]-[13]. These SP sensors are based on the enhanced transmission through arrays of nanoholes. It is a general consensus that these sensors are based on SPRs, while the particular nature of the SPR and the mechanism behind the sensing is still a questing open for discussion. In this paper, we will theoretically study the transmission through arrays of nanoholes and point out the contribution for sensing from the localized waveguide resonant mode utilizing the rigid full-vectorial three-dimensional (3D) finite-difference time-domain (FDTD) method.

\section{CALCULATIONS AND DISCUSSION}

Consider a gold $(\mathrm{Au})$ film patterned with a periodic square array of rectangular air holes. The Au film, assumed to be on a glass substrate $(\varepsilon=2.117)$, has a thickness of $h=200 \mathrm{~nm}$ in the $\mathrm{z}$ direction, the dimensions of the holes in $\mathrm{xy}$ directions are denoted by $\mathrm{Lx} \times \mathrm{Ly}$, and the lattice constant is denoted by L. The insets in Figure 1 show the corresponding structure with cross sections in the xy and yz planes, where the green region represents the substrate while red for the metal. For a similar structure, it has been pointed out $[8,9]$ that there are two different resonances attributing to the enhanced transmission: (i) localized waveguide resonances where each air hole can be considered as a low-quality-factor resonator, and (ii) well-recognized surface plasmon resonances due to the periodicity. These results have been explained well by the band structure theory [9]. To further support these explanation, here we study the transmission through the $\mathrm{Au}$ film with periodic square arrays of different air-holes shape. Consider the case of normal incidence, and the electric field of the incident wave is polarized along the short edge of the rectangular holes (the $x$ direction). Transmission through the film is calculated by the time-domain auxiliary differential equation approach combining 3D FDTD models of a dispersive material [14, 15]. The dielectric function of $\mathrm{Au}$ is described by the lossy Drude model

$$
\varepsilon(\omega)=\varepsilon_{\infty}-\frac{\left(\varepsilon_{0}-\varepsilon_{\infty}\right) \omega_{p}^{2}}{\omega^{2}+2 i \omega \nu_{c}},
$$

where $\varepsilon_{\infty}$ and $\varepsilon_{0}$ are the relative permittivities at infinite and zero frequency, respectively, $\omega_{p}$ is the plasma frequency, and 
$v_{c}$ is the collision frequency. We choose $\varepsilon_{\infty}=12.18, \varepsilon_{0}=12.75$, $\omega_{p}=1.916 \times 10^{16} \mathrm{rad} / \mathrm{s}$, and $v_{c}=1.406 \times 10^{14} \mathrm{rad} / \mathrm{s}$ for the Drude model, which fits the experimental data [16] quite well.

Figure 1 shows the transmission through the gold film with a square array of rectangular holes with different sizes in the xy directions. The peak in the transmission at the wavelength $\lambda=617 \mathrm{~nm}$ hardly moves with varying the air-holes shape, while, the position of another peak is strongly dependant on the air-holes shape.

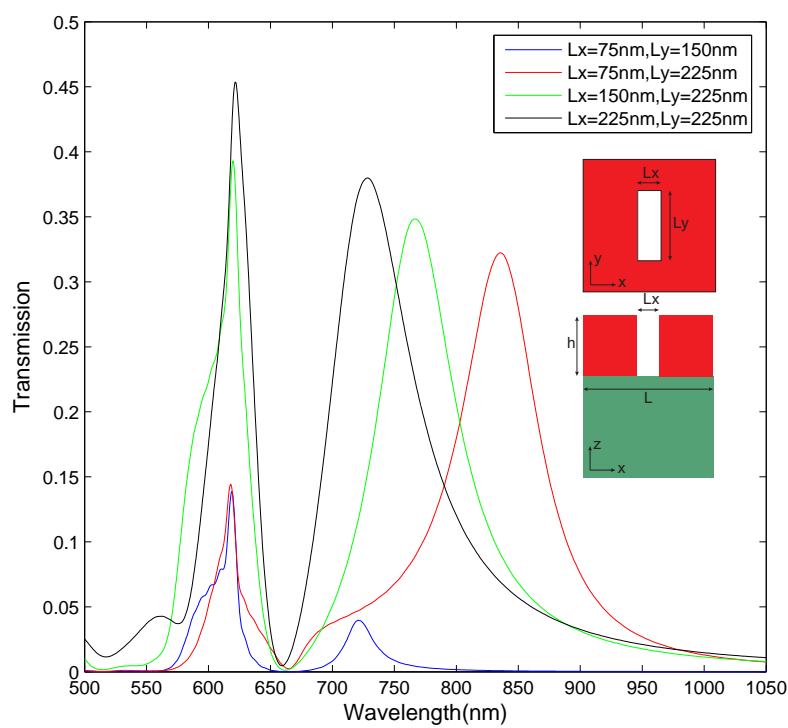

FIG. 1 Transmission through the Au films with periodic square arrays of aperture with different air-holes shape. The Au film is on a glass substrate. The dimension of the holes is denoted by Lx $\times$ Ly, the thickness of the film is $200 \mathrm{~nm}$ and the lattice constant (L) is $425 \mathrm{~nm}$.

For normally incident light through arrays of sub-wavelength holes, to a good approximation the resonance wavelengths are given by a standing-wave quantization of the surface plasmon dispersion for a smooth film at normal incidence [1, 2],

$$
\lambda_{\text {max }}^{S P}(i, j)=\frac{L}{\sqrt{i^{2}+j^{2}}} \sqrt{\frac{\varepsilon_{1} \varepsilon_{2}}{\varepsilon_{1}+\varepsilon_{2}}} .
$$

Here, $\mathrm{L}$ is the periodicity of the array, $\varepsilon_{1}$ is the dielectric constant of the interface medium, $\varepsilon_{2}$ is that of the metal, and $i$ and $j$ are integers defining the different diffraction orders. Certainly, each interface can sustain SPs and the transmission spectra contain two sets of peaks associated with each surface. In accordance with Eq. (2), we conclude that the peak around $617 \mathrm{~nm}$ in Figure 1, due to periodic surface plasmon wave, is related to the $(1,0)$ Au-glass resonance. For the Au-air resonant modes, the resonant wavelengths are always less than $425 \mathrm{~nm}$, which are not considered in this paper. The peak related to the surface plasmon mode, hardly shifts when varying the size of the air holes, which can be explained well by Eq. (2). On the other hand, another resonant mode, corresponding to the localized waveguide resonant mode significantly depends on the hole size, which is naturally understood by the resonant condition. Both results shown in Figure 1 coincide with those mentioned in Ref. [9], which gives another explanation for the mechanism of the enhanced transmission.
As mentioned above, the response of the SP is very sensitive to the refractive index in the vicinity of the metal surface. Hence, simply placing a thin layer on a hole array will shift the position of its transmission peaks. Consider a structure with yz cross section shown in the inset of Figure 2. The dimensions of the holes in $x y$ directions are $225 \times 150 \mathrm{~nm}^{2}$ and the lattice constant is $425 \mathrm{~nm}$. Other parameters correspond to those mentioned in Figure 1. Suppose the metal interface is covered by a uniform layer (blue region), with a height of $w=25 \mathrm{~nm}$, see inset of Figure 2. Figure 2 shows transmission spectra for the Au film being covered by a uniform layer with a refractive index increasing from $n=1.0$ to $n=1.5$ in steps of 0.1 .

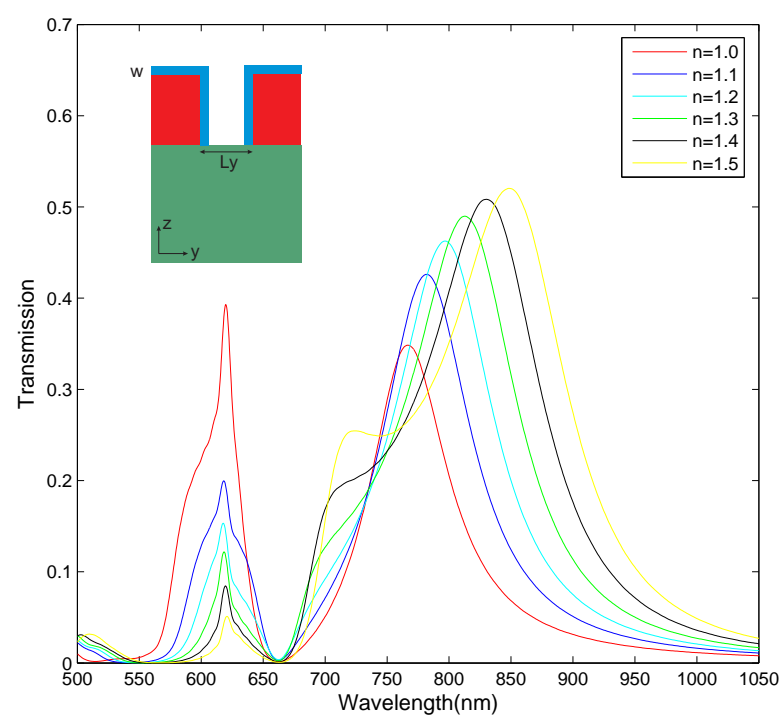

FIG. 2 Transmission through the Au films with periodic arrays of nanoholes being coated by different materials with refractive indices varying from $n=1.0$ to 1.5 in steps of 0.1 .

One can see clearly that the peak, around $617 \mathrm{~nm}$, does not shift when varying the refractive index of the attached layer. As seen from Eq. (2), the resonant peak $(617 \mathrm{~nm})$, related to the $(1,0)$ Au-glass resonance, only depends on the lattice constant and the effective refractive index at the metal-glass interface. Obviously, it is independent on the coverage of the Au-air interface, i.e. this resonant mode is not a candidate for a SPR sensor. We emphasize that the simulation results agree well with theoretical ones obtained from Eq. (2). It should be noted that the surface plasmon resonant modes on the Au-air surface depend on the refractive index of the layer attached to the surface, so that the SPR can be used as a sensor. However, in this paper, we do not consider these modes since they fall outside our wavelength region of interest. On the other hand, peaks, corresponding to the localized waveguide modes, significantly shift due to the change of the refractive index of the layer. As mentioned above, each air hole can be considered to be a section of metallic waveguide with both ends open to free space, forming a low-quality-factor resonator. When varying the effective refractive index of the layer in air holes, the resonant condition changes and the corresponding peaks obviously shift. The peak related to the localized waveguide mode shifts $\delta \lambda=16 \mathrm{~nm}$ when the surface is modulated from $n=1.0$ to $n=1.1$, thus giving potential for sensor applications. When 
we further increase the height of the layer, the peak related to the periodic surface plasmon wave hardly shifts while the peak for the localized waveguide mode is strongly sensitive to the refractive index of the layer. The sensitivity becomes larger when increasing the height of the layer. As an example, the shift becomes $\delta \lambda=18 \mathrm{~nm}$ for the case of $w=50 \mathrm{~nm}$ as shown in Figure 3, when the surface is modified from $n=1.0$ to $n=1$.1. It was always believed that only periodic surface plasmon resonant mode contributes to sensing for the SPR based sensor. In this paper, we emphasize the contribution for sensing from the localized waveguide mode, although the detected peak position change is not significant and the peak is quite broad. This may pave a new way to design SPR based sensors.

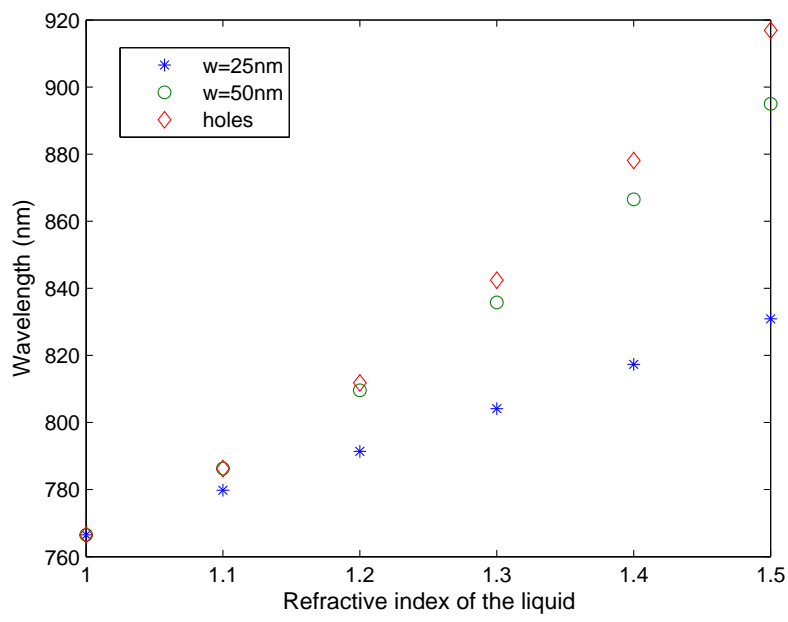

FIG. 3 Wavelength shift as a function of the refractive index of the material.

The analysis above demonstrates that the localized waveguide mode takes an important role for the SPR based sensor. Furthermore, we recently found that for frequencyindependent dielectric-function structure the sensitivity is proportional to the filling factor $f$, defined by $f=\langle E \mid D\rangle_{1} /\langle E \mid D\rangle$ [17]. The integral in the numerator of the filling factor is restricted to the region containing the fluid, or in the present case the dielectric layer, while the other integral is over all space. The periodic surface plasmon mode shown above is a $(1,0)$ Au-glass resonance mode, where most of the energy is believed to be bound at the Au-glass surface. Therefore, $f$ is close to zero, i.e. the peak related to this mode will not shift when the Au-air surface is modified, which is in agreement with the calculated result in Figure 2. To increase the sensitivity, we further consider the structure with air holes being filled with dielectric media (blue region) as shown in the inset of Figure 4. Other parameters correspond to those in Figure 2. Figure 4 shows transmission spectra for the $\mathrm{Au}$ film with air holes being filled by different materials with the refractive index increasing from $n=1.0$ to $n=1.5$ in steps of 0.1. Similar to the result in Figure 2, the peak around $617 \mathrm{~nm}$ in Figure 4 does not shift when varying the refractive index of the media in the air holes, which agrees well with that obtained from Eq. (2). On the other hand, the peak related to the localized waveguide mode significantly shifts due to the change of the refractive index of the media. As mentioned above, each air hole can be considered to be a low-quality-factor resonator. When varying the refractive index of the media in the air holes, obviously the peak does shift. Compared to the result shown in Figure 2, the sensitivity becomes better as shown in Figure 3. The shift for the localized waveguide mode is around $\delta \lambda=19 \mathrm{~nm}$ when the air holes (with index $n=1$ ) are filled by a m3dia of index $n=1.1$. Due to the low quality-factor, $f$ will never be close to unity. If the quality factor of the waveguide resonator can be increased, we believe that the sensitivity will become stronger since $f$ will become larger. We note that another peak starts to appear when the refractive index increases above 1.3, which is also dependant on the refractive index of the medias as shown in Figure 4. Quite naturally, the high-order mode appears, corresponding to a high-order resonant waveguide mode appearing in a cavity composed by metallic waveguide when increasing of the refractive index of the material inside the cavity.

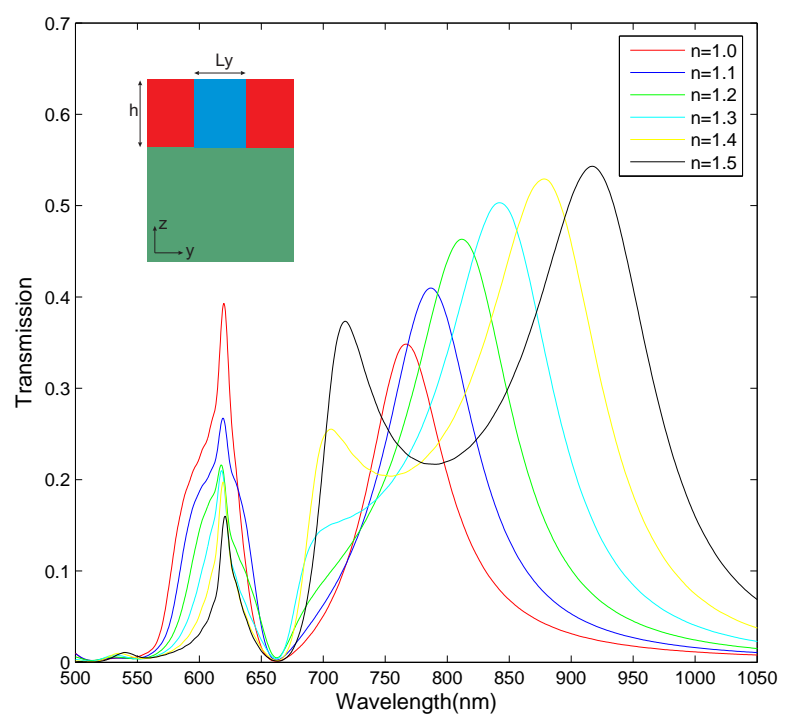

FIG. 4 Transmission through the Au films with periodic arrays of nanoholes being filled by different medias with refractive indices varying from $n=1.0$ to 1.5 in steps of 0.1 .

\section{CONCLUDING REMARKS}

In this paper, we have studied transmission through arrays of subwavelength holes in gold films utilizing the rigid full-vectorial three-dimensional finite-difference time-domain method. Based on the two different resonance mechanism for the enhanced transmission, our calculations show that in our wavelength range of interest, the localized waveguide resonant mode attributes to sensing rather than periodic goldglass surface plasmon resonant modes. It was believed that only periodic surface plasmon resonant mode contributes for sensing for the SPR based sensor. In this paper, we emphasize the contribution for sensing from the localized waveguide mode. Although the detected peak is fairly broad and the shift is not too pronounced, this may pave a new way to design of SPR based sensors. 


\section{ACKNOWLEDGMENTS}

This work is financially supported by the Danish Council for Strategic Research through the Strategic Program for Young Researchers (grant no: 2117-05-0037). M. Qiu acknowledges the support from the Swedish Foundation for Strategic Research (SSF) on INGVAR program, the SSF Strategic Research Center in Photonics, and the Swedish Research Council (VR).

\section{References}

[1] T. W. Ebbesen, H. J. Lezec, H. F. Chaemi, T. Thio, and P. A. Wolff, "Extraordinary optical transmission through sub-wavelength hole arrays" Nature 391, 667-669 (1998).

[2] H. F. Ghaemi, T. Thio, D. E. Grupp, T. W. Ebbesen, and H. J. Lezec, "Surface plasmons enhance optical transmission through subwavelength holes" Phys. Rev. B 58, 6779-6782 (1998).

[3] J. A. Porto, F. J. Garcia-Vidal, and P. B. Pendry, "Transmission resonances on metallic gratings with very narrow slits" Phys. Rev. Lett. 83, 2845-2853 (1999).

[4] L. Martin-Moreno, F. J. García-Vidal, H. J. Lezec, K. M. Pellerin, T. Thio, J. B. Pendry, and T. W. Ebbesen, "Theory of extraordinary optical transmission through subwavelength hole arrays" Phy. Rev. Lett. 86, 1114-1117 (2001).

[5] A. Krishnan, T. Thio, T. J. Kim, H. J. Lezec, T. W. Ebbesen, P. A. Wolff, J. B. Pendry, L. Martin-Moreno, and F. J. Garcia-Vidal, "Evanescently coupled resonance in surface plasmon enhanced transmission" Opt. Commun. 200, 1-7 (2001).

[6] K. J. Klein Koerkamp, S. Enoch, F. B. Segerink, N. F. van Hulst, and L. Kuipers, "Strong influence of hole shape on extraordinary transmission through periodic arrays of subwavelength holes" Phys. Rev. Lett. 92, 183901 (2004).
[7] K. L. van der Molen, K. J. Klein Koerkamp, S. Enoch, F. B. Segerink, N. F. van Hulst, and L. Kuipers, "Role of shape and localized resonances in extraordinary transmission through periodic arrays of subwavelength holes: Experiment and theory" Phys. Rev. B 72, 045421 (2005).

[8] F. J. Garcia-Vidal, E. Moreno, J. A. Porto, and L. Martin-Moreno, "Transmission of light through a single rectangular hole" Phys. Rev. Lett. 95, 103901 (2005).

[9] Z. Ruan and M. Qiu, "Enhanced transmission through periodic arrays of subwavelength holes: The role of localized waveguide resonances" Phys. Rev. Lett. 96, 233901 (2006).

[10] J. Homola, S. Yee, and G. Gauglitz, "Surface plasmon resonance sensors: review" Sensor Actuat. B:Chem. 54, 3-15 (1999).

[11] A. G. Brolo, R. Gordon, B. Leathem, and K. L. Kavanagh, "Surface plasmon sensor based on the enhanced light transmission through arrays of nanoholes in gold films" Langmuir 20, 48134815 (2004).

[12] T. Rindzevicius, Y. Alaverdyan, A. Dahlin, F. Hook, D. S. Sutherland, and $\mathrm{M}$. Kall, "Plasmonic sensing characteristics of single nanometric holes" Nano Lett. 5, $2335-2339$ (2005).

[13] K. A. Tetz, L. Pang, and Y. Fainman, "High-resolution surface plasmon resonance sensor based on linewidth-optimized nanohole array transmittance" Opt. Lett. 31, 1528 - 1530 (2006).

[14] A. Taflove, Computational Electrodynamics: The Finite-Difference Time-Domain Method (2 edn. Artech House INC, Norwood, 2000).

[15] S. Xiao, L. Liu, and M. Qiu, "Resonator channel drop filters in a plasmon-polaritons metal" Opt. Express 14, 2932-2937 (2006).

[16] E. D. Palik, Handbook of Optical Constants of Solids (Academic, New York, 1985).

[17] S. Xiao and N. A. Mortensen, "Highly dispersive photonic bandgap-edge optofluidic biosensors" JEOS:RP. 1, 06026 (2006). 